\title{
LA PERSONA HUMANA EN SAN BUENAVENTURA
}

\author{
Manuel Lázaro Pulido \\ Instituto Teológico «San Pedro de Alcántara» (Cáceres)
}

\begin{abstract}
RESUMEN
San Buenaventura elabora el concepto de persona humana a partir de dos contextos. Uno más general, que es el ejemplarismo, el cual explica las relaciones existentes entre Dios y las creaturas en términos de participación metafísica y simbólica. El otro, de carácter teológico, responde a la cuestión trinitaria y cristológica, y le lleva a plantearse el concepto de «persona». La estructura humana queda determinada por su carácter personal. San Buenaventura utiliza la analogía para hablar de la persona creada, que define en términos de relación, individuación y dignidad. De esta forma desarrolla el sentido de persona, presente en las Sagradas Escrituras.
\end{abstract}

Palabras clave: Buenaventura (san), ejemplarismo, individuo, persona, relación.

\begin{abstract}
Saint Bonaventure elaborates the concept of human person starting from two contexts. One, more general: the exemplarism, which explains the relationships between God and creatures in de manner of metaphysical and symbolic participation. The other, of teheological characteristic, is an answer to the Trinitarian an christological question and it makes him to ask himself the concept of person. The human structure is definitively determinated by its personal characteristic. Saint Bonaventure uses the analogy in order to speak of the created person, whom defines as relationships, individuation and dignity. And in this manner he develops the biblical meaning of person.
\end{abstract}

Key words: Bonaventure (saint), exemplarism, individuation, person, relationship

\section{INTRODUCCIÓN: BASE EJEMPLARISTA DE LA METAFÍSICA EXPRESIVA EN EL HOMBRE COMO PERSONA}

La persona humana en san Buenaventura se desarrolla a partir de un contexto de pensamiento que le lleva a realizar una antropología metafísica en la que la delimitación del ser queda absorbida por el dinamismo del ser que «es» siendo presencia. De una parte, ha de tenerse en cuenta una tradición metafísica de gran calado que tiene como fuente la lectura medieval de Platón mediante el neoplatonismo, bifurcada en dos caminos (ejemplarismo agustiniano y simbolismo dionisiano) y que resuelve cruzarse en el siglo XII en la abadía de Saint-Victor. ${ }^{1}$ De otra parte, una tradición teológica alimentada en las interpretaciones y glosas del texto de la Escritura $(\mathrm{Gn} 1,26)$ que consagra en occidente, desde san Agustín, la teología de la imagen en la naturaleza, en especial en el hombre, capaz de recibir mediante la gracia divina la facultad de similitud con el Dios Trino (imago in na-

1 Cf. Zas Friz de Col, R., La teología del símbolo de san Buenaventura, Roma, Ed. Pontificia Università Gregoriana, 1997, pp. 63-137; Lázaro, M., La creación en Buenaventura: Acercamiento filosófico a la metafísica expresiva del ser finito, Grottaferrata (Roma), Frati Editori di Quaracchi, 2005, pp. 61-101. 
turalibus, similitudo in gratuitis), alimentada por la teología del siglo XII y los primeros maestros parisinos. ${ }^{2}$ Por último, la fuente franciscana, de modo especial la persona de san Francisco de Asís, que es el modelo personal de imagen humana en su naturaleza y de semejanza con Dios dada por la gracia, ejemplo vivo de la hermenéutica existencial y recapitulativa (vir hierarchicus). ${ }^{3}$

A partir de estos elementos de influencia se desarrolla una metafísica del ser creado que se caracteriza por su carácter relacional. El ser creado expresa su ser participado. Como telón de fondo vital está presente la actitud de san Francisco ante la creación, su armonía y belleza, y como perspectiva filosófico-teológica, el ejemplarismo agustiniano desarrollado por el Seráfico.

Efectivamente, la pregunta por el concepto de persona en san Buenaventura tiene desde el punto de vista filosófico una fuente especial en la doctrina del ejemplarismo del que es un exponente, que podríamos denominar, acabado. Pero este ejemplarismo que incluye todo el orden de lo real, desde el macrocosmos hasta el microcosmos en cuanto que se puede definir como «la doctrina de las relaciones de expresión existentes entre las creaturas tal como son en sí mismas y tal como son en Dios o en el Verbo», ${ }^{4}$ cobra en el pensamiento antropológico una orientación que en términos más actuales podríamos llamar personalista. ${ }^{5}$ El concepto de persona aplicado al hombre recibe un dinamismo muy especial sobre el eje de la concepción humana de imagen y semejanza de Dios. Es necesario fijarse, aunque sea brevemente, en la perspectiva ejemplarista que constituye la realidad en la que es comprendida el ser humano para poder explicar el hombre en cuanto persona, aparte de este mismo concepto tomado de la doctrina trinitaria.

En su realidad metafísica el hombre que es libre está siempre referido a Dios que nos ha creado libremente. La libertad del acto creador en su producción y en su obra no borra la huella que ha dejado en nosotros como creación suya. Como sintetiza con gran acierto Bernardino de Armellada: «Dios creador de las cosas, que se ha revelado en Cristo como trino en Personas, tuvo que dejar su huella en las criaturas (...) Buenaventura, desde esta ejemplaridad de Dios y con su profundidad de teólogo místico, detecta la huella de Dios Trino en todas las dimensiones de la creación desde los seres inanimados hasta el mismo discurrir de la historia». ${ }^{6}$ De este modo, en la raíz de la concepción personal en el hombre anida la idea ejemplar de la realidad natural como espejo de Dios. Todos los seres creados participan del hecho de la creación, pero no del mismo modo. La diferencia entre ellos implica una distinción en la representación del Creador, por lo que los seres lo son en una gradación expresiva ascendente (som-

2 Chavero, F. de A., «Imago creationis, imago recreationis. Para una teología y antropología de la imagen de Dios en san Buenaventura», en Carthaginensia, 9 (1993), p. 81. Cf. Javelet, R., Image et ressemblance au douzième siècle: De Saint Anselme à Alain de Lille, 2 vols., Paris, Letouzey et Ané, 1967.

3 Cf. Hayes, Z., «The theological image of St. Francis of Assisi in the sermons of St. Bonaventure», en Chavero, F. de A. (ed.), Bonaventuriana: Miscellanea in onore di Jacques Guy Bougerol ofm. Vol. 1. Roma, Ed. Antonianum, 1988, pp. 323-321; Chavero, F. de A., «Vir hierarchicus (Leganda maior, Prologus), ¿Una interpretación de san Francisco en clave dionisiana?», en Il Santo, 40 (2000), pp. 7-48.

4 Bissen, J. -M., L'exemplarisme divin selon Saint Bonaventure, Paris, Vrin, 1929, p. 4. Sobre el ejemplarismo cf. Oromí, M., «Filosofía ejemplarista de San Buenaventura, Introducción general», en Obras de san Buenaventura, vol. 3, Madrid, BAC, 1957, pp. 3-138; Manferdini, T., «L'exemplarismo in san Bonaventura», en Incontri Bonaventiriani, 7 (1972), pp. 41-80; Valderrama, C., «Sentido de la filosofía a la luz del ejemplarismo bonaventuriano», en Bolívar, 16-20 (1953), pp. 963-972; Id., «Filosofía ejemplarista. Acercamiento al pensamiento de San Buenaventura», en Franciscanum, 16 (1974), pp. 160-468; Javelet, R., «Réflexions sur l'exemplarisme bonaventurien», en Comissionis Internationalis Bonaventurianae, San Bonaventura 1274-1974 Volumen commemorativum anni speties centenarii a morte S. Bonaventurae Doctoris Seraphici, vol. 4, Grottaferrara (Roma), Collegio S. Bonaventura, 1974, pp. 349-370.

5 Chavero, F. de A., Imago Dei. Aproximación a la antropología teológica de san Buenaventura, Murcia, Espigas-Instituto Teológico Franciscano, 1993, p. 225.

6 Armellada, B. de, «Antropología teológica. Creación, pecado, gracia, escatología», en Merino, J. A., y Martínez, F. (coord.), Manual de Teología franciscana, Madrid, BAC, 2003, p. 373. 
bra, vestigio, imagen y similitud), siendo el hombre cumbre de los seres finitos y creados: el hombre es verdadera imagen de Dios, porque es similitud expresa. ${ }^{7}$

El Doctor Seráfico describe el hombre, a partir de una lectura filosófico-teológica de la Escritura (Gn 1, 26), como un verdadero ser comunicativo que tiene la capacidad de alcanzar distintos grados de mostración y significación ontológica; desde el modo más lejano de vestigio, en virtud de su naturaleza, al modo más próximo de imagen de Dios, en virtud de su alma (mens). Su constitución expresiva afecta a su ser personal en cuanto que como imagen queda abierto al Otro, al horizonte trascendente, estando capacitado, por gracia, a mantener semejanza con Él. El hombre no puede ser definido sólo en virtud de una unión sustancial de cuerpo y entendimiento o razón, sino que exige una explicación que rompa con las fronteras de su propia individualidad, sin negarla, muy al contrario fundamentándola. El ser humano ha de poder expresarse en términos de relación, pues ser imagen es ser representación, y ello significa que es «siempre correlativo a un prototipo, a un ejemplar que explica». ${ }^{8}$ La categoría antropológica de la relación a Dios, a los demás hombres y al mundo, se explica desde la utilización del maestro franciscano de la metafísica ejemplarista, la causa ejemplar, y la metafísica emanantista. ${ }^{9}$ Con este arsenal puede explicar el significado expresivo del hombre y la creación. El hombre en su constitución es expresión de forma pasiva de la realidad divina, está abierto al misterio Trinitario. Y Dios Trinitario, desde la expresividad activa, hace posible la constitución expresiva antropológica. ${ }^{10} \mathrm{El}$ hombre en virtud de su propia constitución creada a imagen y semejanza del Creador es un ser abierto desde lo más íntimo de su ser al mundo.

Resumiendo, el ejemplarismo es la doctrina que ilumina y acompaña la noción de persona. Explica la utilización de la categoría «persona» en el hombre a partir de su explicación en la Trinidad. El efecto del acto creador de Dios Creador Uno y Trino, explicado en lenguaje filosófico bajo los nombres de causa eficiente, ejemplar y final, es punto clave de entendimiento de toda la realidad creadora, corporal o espiritual en su conjunto, y especialmente del hombre, de naturaleza corporal y espiritual, quien en cuanto imagen y semejanza refleja, aún de modo imperfecto, su fundamento. ${ }^{11}$

\section{EL CONCEPTO DE PERSONA}

Todo lo que hemos dicho hasta ahora tiene una repercusión orientadora de la especulación sobre la persona en el hombre. La metafísica de la expresión, de comunicación lleva al hombre a la interioridad. Una lectura en clave antropológica del Itinerarium nos mostraría cómo el ser humano se define por nacer con vocación de apertura y de continua proyección.

7 «Homo est vere imago Dei, quia est eius expressa similitudo» (S. Buenaventura, II Sent., d. 16, a. 1, q. 1 concl.: II, 394).

8 Mathieu, L., La Trinité créatrice d'après saint Bonaventure, Paris, Ed. Franciscaines, 1992, p. 230.

9 Sobre la dualidad de fuentes en la explicación metafísica expresiva cf. Lázaro, M., «La creación en Buenaventura», o. c., pp. 61-101.

10 «(..) sicut exemplar secundum proprietatem vocabuli dicit expressionem per modum activi, —unde exemplar dicitur ad cujus imitationem fit aliquid-, sic e contrario imago per modum passivi; et dicitur imago quod alterum exprimit et imitator» (S. Buenaventura, I Sent., d. 31, p. 2, a. 1, q. 1 concl: I, 540). Sobre el aspecto pasivo y activo del ejemplarismo cf. Bougerol, J.-G., Introduction à saint Bonaventure, París, Vrin, 1988, p. 77.

11 Un texto sintético y maduro donde explica la creación del mundo es el siguiente: «Per hoc autem, quod additur in certo pondere, numero et mensura, ostenditur, quod creatura est effectus Trinitatis creantis sub triplici genere causalitatis: efficientis, a quo est in creatura unitas, modus et mensura, exemplaris, a quo est in creatura veritas, species et numerus; finalis, a quo est in creatura bonitas, ordo et pondus. Quae quidem reperiuntur in omnibus creaturis tanquam vestigium Creatoris sive corporalibus, sive spiritualibus, sive ex utrisque compositis» (S. Buenaventura, Brevil., p. 2, c. 1: V, 219). 
El hombre es un ser abierto, en permanente peregrinaje por el camino de la vida en el mundo y en el tiempo. Frente a la cerrazón de la lectura subjetivista, el hombre encuentra su ser en un itinerario interior que le lleva a abrirse al Otro, encontrando su sustancia - lo que es, su realidad - en la similitud con Dios. Esta certeza del ser creado es analogía de la realidad divina. El ser humano como persona está abierto a la relación, conjugando individualidad esencial con relación. Por lo tanto, el carácter aperturista de la metafísica influye en la articulación de su pensamiento especulativo filosófico-teológico y, consiguientemente, en su concepto de persona. ${ }^{12}$

Ya que la antropología bonaventuriana se explica desde los parámetros de la metafísica ejemplarista, es lógico que el análisis del concepto de persona y su constitución ontológica los aborde respecto de la Trinidad (I Sent, d. 25) y la naturaleza de los ángeles (I Sent., d. 33-34). Esto no significa ni mucho menos que no pueda hablarse con propiedad de la persona humana en san Buenaventura. Como hemos mencionado, a su conocida doctrina ejemplarista ha de sumarse la categoría personalista que fundamenta el carácter relacional del hombre, siendo uno de los fundamentadores del personalismo como expresión de la interpretación y propuesta cristiana del hombre. Como afirmó É Gilson, el Maestro franciscano «parece haber estado particularmente feliz en su análisis de la noción de persona, y es ciertamente uno de los dos o tres mejores intérpretes del personalismo cristiano». ${ }^{13}$ San Buenaventura aplica la noción de persona al hombre, de modo análogo a como se hace en Dios. ${ }^{14}$

Comencemos por ver cómo entiende el concepto de persona y luego cómo se aplica al hombre. El Doctor Seráfico realiza un análisis de la noción de persona recordando distintas definiciones dadas por los teólogos y la Escolástica desde la explicación trinitaria y cristológica. En el concepto de persona aparecen dos concepciones contrapuestas: una apuesta por definir la persona (divina) según la sustancia y otra según la relación. ${ }^{15}$

Tenemos argumentos de autoridad que nos hacen ver que la persona se define por ser sustancia. ${ }^{16}$ El primero en ser citado es el gran referente de la filosofía medieval: el maestro san Agustín. El obispo de Hipona realiza una tentativa de aprehensión de la intimidad personal desde la relación sustancial ${ }^{17}$ que se bifurca en la elaboración medieval en dos tendencias hacia los dos términos.

De un modo más explícito sobre la sustancia se expresa Boecio, en una definición que había sido recogida como un axioma en la época medieval. La enunciación categoriza la persona desde la mirada aristotélica como una «sustancia individual de naturaleza racional». ${ }^{18}$

Junto a estos dos autores de referencia, también cita san Buenaventura a san Anselmo ( Quot sunt personae, tot sunt substantiae») $)^{19}$ o Hugo de San Víctor ( Unum solum nomen est

12 Cf. Iammarrone, L., «La Trinidad», en Merino, J. A., y Martínez, F. (coord.), «Manual de Teología», o. c., pp. $57-148$.

13 Gilson, É., L'esprit de la philosophie médiévale, 2ª Ed., Paris, Vrin, 1998, p. 206, n. 2.

14 S. Buenaventura, I Sent., d. 25, a. 2, q. 2 concl.: I, 444-445.

15 «Utrum nomen persona in divinis dicatur secundum substantiam, an secundum relationem» (S. Buenaventura, Ibid., d. 25 , a. 1 , q. 1 concl.: I, 435ss.).

16 Ibíd., d. 25, a. 1, q. 1: I. 435.

17 «(..) cum dicimus personam Patris, aliud dicimus quam substantiam Patris... Et quemadmodum hoc illi est esse quod Deum esse, quod magnum, quod bonum esse; ita hoc illi est esse, quod personam esse» (S. Agustín, De Trinitate, lib. 7, c. 6, n. 11, PL 42, p. 943).

18 «Persona est naturae rationalis individua substantia» (Boecio, De persona et naturis duabus, c. 3, PL 64, p. 1343c-d). Cf. Lutz-Bachmann, M., " 'Natur' und 'Person' in den 'Opuscula Sacra' des A. M. S. Boethius», en Theologie und Philosophie, 58 (1983), pp. 48-70.

19 « (...) quot sunt personae: quod in pluribus hominibus, qui quot personae, tot individuae sunt substantiae, cognoscitur» (S. Anselmo, Monologion, c. 78, PL 158, p. 221d). 
substantiale, quod de singulis dicitur singulariter, in summa tamen non dicitur singulariter, sed pluraliter pronuntiatur, ut persona: ergo hon nomen persona est nomen substantiales»). ${ }^{20}$

La sustancialidad expuesta, sobre todo por Boecio, no es rechazada de plano por el Seráfico doctor, que si bien no ve inconveniente mayor en la utilización respecto de la persona divina, ve en ella, sin embargo, el peligro de una cosificación de la naturaleza personal, pues ni Dios ni la persona divina son definibles, si bien es posible conocer la persona y comunicarla. En este sentido, su conveniencia no viene de la propia naturaleza a la que se hace referencia cuando hablamos de sustancia de algo, sobre todo, si es Dios; sino que más bien es una utilización que resulta necesaria en virtud de su utilidad, ya que el hombre, limitado, necesita de esta noción para poder acercarse al concepto de persona. Por lo tanto, referirse a sustancia responde más a la imperfección de nuestro entendimiento que a la propiedad del ser divino. ${ }^{21}$ Pero aún así, conviene menos al ser creado, pues puede dar la impresión de dotarle de una carga ontológica propia. Aquí san Buenaventura choca, como pasara a menudo, con aspectos materiales de la doctrina de Aristóteles. No es que desdeñe la doctrina aristotélica, que utilizó sobre todo en su época más joven (como, por ejemplo, la teoría hilemórfica), sino que tiene que ser utilizada desde otros esquemas; de modo que si choca su lectura directa con un entendimiento adecuado de lo que ha de significar ser persona al exagerar la importancia de la categoría de materia en la misma, entonces es mejor realizar una lectura indirecta (in obliquo). ${ }^{22}$

Frente a la apuesta por el polo sustancial como elemento central de la definición de persona, están quienes eligen la relación. Así, Ricardo de San Víctor inclina su captación de la persona por el polo de la existencia, hacia la relación: la persona «es existencia individual de naturaleza racional» 0 «existencia incomunicable». ${ }^{23}$

Las dos tradiciones señaladas habían sido recogidas por el maestro Alejandro de Hales («La persona divina es la hipóstasis diferenciada por la propiedad») ${ }^{24} \mathrm{y}$ transmitidas a su discípulo san Buenaventura, quien integra las diferentes características pertinentes al concepto de persona. La dualidad de fuentes no le plantea ningún problema, sino que, como en otras ocasiones, ${ }^{25}$ realiza una síntesis en la que el dinamismo del concepto sale beneficiado. ${ }^{26}$

20 «Ad se quippe dicitur persona, non ad Filium, vel ad Spiritum sanctum sicut Deus et similia. Hoc enim solum nomen est quod cum dicatur de singulis ad se, pluraliter non singulariter accipiatur in summa. Dicimus namque quod Pater est persona, et Filius persona, et Spiritus sanctus persona. Pater tamen et Filius et Spiritus sanctus non una persona, sed tres» (H. de San Víctor, De sacramentis, p. 1, lib. 2, c. 4, PL 176, p. 377d).

21 S. Buenaventura, I Sent., d. 23, a.1, q. 2 concl.: I, 407.

22 «(..) rationalis naturae cadit in obliquo» (Ibíd., d. 25, a.1, q. 2 concl. ad 2: I, 440).

23 «(...) creata persona est rationalis naturae individua substantia, tam verum est quod quaelibet persona increata est rationalis naturae individua existential» (R. de San Víctor, De Trinitate, lib. 4, c. 23, PL 196, p. 946a) «(...) quod persona divina sit divinae naturae incommunicabilis existential» (Ibíd., lib. 4, c. 22, PL 196, p. 945c) «Fortassis erit planius et ad intelligendum expeditius, si dicimus quod persona sit existens per se solum juxta singularem quemdam rationalis existentiae modum» (Ibíd., lib. 4, c. 24, PL 196, p. 946c). Cf. Reinhardt, E., «La metafísica de la persona en Ricardo de San Víctor», en Soto $\mathrm{M}^{\mathbf{a}} \mathbf{J}$. (ed.), Metafísica y antropología en el siglo $X I I$, Barañáin (Navarra), Eunsa, 2005, pp. 211-230.

24 A. de Hales, Summa Halensis, n. 404: I, 593. Cf. Hufnagel, A., «Die Wesensbestimmung der Person bei Alexander von Hales», en Freiburger Zeitschrift für Philosophie und Theologie, 4 (1957), pp. 148-174; Gunten, A. F. von, «La notion de personne dans la Trinité d'après Alexandre de Halès», en Divus Thomas, 28, (1950).

25 Esta contraposición de fuentes en san Buenaventura referido al concepto de «naturaleza» en Rivera de Ventosa, E., «Doble fuente histórica del concepto de naturaleza en San Buenaventura», en AA. VV., La filosofia della natura nel medioevo: Atti del terzo Congreso internazionale di filosofia medioevale, Passo della Mendola (Trento), 31 agosto - 5 settembre 1964, Milano, Società editrice Vita e pensiero, 1966, pp. 447-454

26 Sobre el concepto de persona en san Buenaventura, cf. Garrido, J. $\mathbf{M}^{\mathbf{a}}$, «El concepto de persona en los textos cristológicos de san Buenaventura», en Verdad y Vida, 22 (1964), pp. 43-75; Id., «Dignidad ontológica de la persona humana en San Buenaventura», en Verdad y Vida, 28 (1970), pp. 283-338; Manzano, I. G., «Concepto de persona humana según S. Buenaventura: una valoración actual de su pensamiento», en Chavero, F. de A. (ed.), «Bonaventuriana», o. c., pp. 391-416. 
Por último, en otro pasaje posterior donde está analizando la definición de Boecio poniéndola en relación con la de Ricardo de San Víctor, presenta otra definición de persona propuesta por los maestros y que cita explícitamente: la persona queda definida como «hipóstasis distinguida por una propiedad perteneciente a la dignidad». ${ }^{27}$ De las definiciones de Boecio y de los maestros se toma la parte común, sustancial, de la persona (pues decir hipóstasis, es decir sustancia individual); ${ }^{28}$ si bien el primero subraya la razón de la cosa (rei), lo maestros inciden en señalar la razón de su cambio (translationis). La definición de Ricardo, sin sustituir estas propiedades personales, las integra al asignar la propiedad de la incomunicabilidad y la relación como clave de interpretación de las anteriores. De Boecio subraya la sustancia sin darle un protagonismo absoluto; de Ricardo toma la incomunicabilidad.

Teniendo estas fuentes en la cabeza, integra las cualidades de la tradición anterior y las mejora. Su maestro Alejandro de Hales había afirmado que la persona (divina) siendo naturaleza sustancial, implica, en virtud de su realidad, relación, siendo ésta mediación entre la sustancia común y lo predicado de modo relativo. ${ }^{29} \mathrm{El}$ Seráfico prolongando la formulación halesiana define la persona de la siguiente manera: «se dice persona de suyo racional como un supuesto distinguido por una propiedad perteneciente a una dignidad». ${ }^{30}$ Así, afirma, en primer lugar, que entendemos de la persona que es una sustancia; sin embargo, si hacemos caso al verdadero entendimiento vemos que, en segundo lugar, en Dios, persona hace referencia a la relación.

Siguiendo san Buenaventura la definición de Boecio -que como hemos señalado ni mucho menos desdeña, afirmando de ella su bondad y conveniencia-,${ }^{31}$ persona se entiende como principio de acción en sí mismo por ser supuesto y, por lo tanto, constituido del ser sustancial. De ahí, que podemos afirmar que persona designa el supuesto (suppositum) de una naturaleza racional distinguido por una propiedad. ${ }^{32}$

En esta explicación se contienen dos realidades fundamentales en la persona. De un lado, el nombre «persona» alude a la naturaleza común, es decir, a la esencia sustancial, la que responde a la pregunta por el qué de las cosas. Por lo tanto, remite a lo común no en su sentido más general y abstracto (esencia), sino en su concreción al supuesto (sustancia). De otro lado, persona es, también, el supuesto de la distinción de la propiedad. El hecho de tener propiedad le confiere «con propiedad» poseer un ser de relación, pues lo mismo es relación o propiedad. ${ }^{33}$ Consecuentemente, si decimos de la persona que es supuesto distinguido de propiedad, podemos afirmar que lleva consigo tanto el hecho de ser sustancia como relación. Si bien el polo sustancial de la definición posee un carácter primordial en cuanto que ilumina el intelecto sobre la parte común y general, tanto de la persona en Dios como en las creaturas, el tener propiedades diferenciadoras supone acentuar de igual modo y peso el de relación.

La conveniencia de equilibrar los dos puntos en los que se apoya la definición de persona se explica al considerar que un ser abierto a realidades con propiedades libera el concepto de persona de cualquier tentación que, de parte de la definición sustancial, pudiera cosificarla, como indicamos respecto de la definición boeciana. Frente al carácter de «cosa» e «indivi-

27 «A magistris definitur sic: persona est hypostasis distincta proprietate ad nobilitatem pertinente» (S. Buenaventura, I Sent., d. 25, a.1, q. 2 concl. ad. 4: I, 441).

$28 \quad$ Ibíd., d. 25 , a.1, q. 2 concl.: I, 440.

29 A. de Hales, Summa Halensis, n. 386: I, 596.

30 «Respondeo: Dicendum, quod persona de sui ratione dicit suppositum distinctum proprietate ad dignitatem pertinente» (S. Buenaventura, I Sent., d. 23, a. 1, q. 1 concl: I, 405); «Persona dicitur rationalis naturae suppositum proprietate distinctum» (Ibíd., d. 25, a.1, q. 2 concl.: I, 436. También en Ibíd., d. 34, a. un, q. 1 concl. ad. 2 : I, 587).

31 «Definitio illa Boethii est bona et conveniens divinis personis» (Ibíd., d. 25, a.1, q. 2 concl.: I, 439).

32 «ersona dicitur rationalis naturae suppositum proprietate distinctum» (Ibíd., d. 25, a.1, q. 2 concl.: I, 436. También en Ibíd., d. 34, a. un, q. 1 concl.: I, 587).

33 « (...) similiter quaedam relatio est propietas» (Ibíd., d. 26, a. un., q. 4 concl.: 460). 
duo» que la categoría sustancial rememora en términos aristotélicos, se refuerza la idea de comunicación personal. ${ }^{34}$ Pues no hemos de olvidar que no nos interrogamos sobre el qué (la esencia), ni lo qué, lo común a una pluralidad de sujetos (la sustancia), sino sobre el quién (quis est): ${ }^{35}$ sobre la identidad de la cosa o el sujeto dotado de la esencia de modo propio, incomunicable, en cuanto supuesto sustancial distinto (hipóstasis) por una propiedad noble. Persona hace, pues, referencia a lo distinto y a lo concreto. Más tarde en el Breviloquium, una obra de vocación didáctica y científica a la vez, con un estilo «elegante al servicio de un pensamiento claro y profundo», ${ }^{36}$ nos ofrece un resumen de lo aquí dicho:

(...) en Dios la sustancia puede ser significada de diversos modos, a saber: como comunicable y como incomunicable. En cuanto comunicable, en abstracto por el nombre de esencia y en concreto por el nombre de sustancia; mas en cuanto incomunicable, o como distinguible por el nombre de hipóstasis o como distinta por el nombre de persona. $\mathrm{O}$ de otro modo: como distinta de cualquier modo, y así es hipóstasis, o como distinta notable y perfectamente y es persona. Los ejemplos de estos cuatro casos en la creatura son: humanidad, hombre, algún hombre, Pedro. El primer nombre dice la esencia; el segundo, la sustancia; el tercero la hipóstasis y el cuarto, la persona. ${ }^{37}$

De este modo, queda bien clara la diferencia entre la naturaleza y la persona. No olvidemos que la pregunta por la persona surge en un contexto de explicación trinitaria, de ahí, la necesidad de distinguir entre naturaleza y persona. $Y$ así, frente a la naturaleza como «diferencia específica que informa un ser cualquiera» (Buenaventura cita de nuevo a Boecio), afirmamos que «persona es una existencia incomunicable», por lo que «en esto difieren: en que la naturaleza significa la forma misma por la que una cosa es lo que es, mientras que la persona significa el supuesto individual e incomunicable». ${ }^{38}$ Lógicamente, hemos de tener en cuenta que la identidad es de una cosa a lo que responde la sustancia, en cuanto a lo común de la cosa; pero respecto a lo que es propio a la concreción de lo que se es, responde la persona en sí, por lo que no puede identificarse con la sustancia. La persona añade a la naturaleza algo positivo que distingue, no lo que es su realidad - ya que a Dios (objeto propio de la reflexión sobre la persona) que es sustancia simple no se le puede añadir algo como entidad propia—, ${ }^{39}$ sino el cómo es, su modo sustancial. La identidad que corresponde a la persona supone tanto la sustancia como la subsistencia (hipóstasis). Es decir, presume una parte común, comunicable, y una parte propia que es incomunicable:

A lo que se objeta que la persona se define o por la sustancia o por la relación, hay que decir que persona no es otra cosa que una 'hipóstasis, que se distingue por la propiedad'; y por eso se predica en virtud de una relación intrínseca y de la sustancia no común, sino propia. ${ }^{40}$

\footnotetext{
34 Marías, J., Persona, Madrid, Alianza Editorial, 1997, pp. 85-91.

35 S. Buenaventura, I Sent., d. 23, a. 1, q. 3 concl: I, 409. Cf. Ibíd., d. 26, a. un., q. 4 concl.: 461.

36 Bougerol, J. -G., «Introduction», o. c., 196.

37 « (...) ideo necesse est, ibi multipliciter significari substantiam, scilicet ut communicabilem et incommunicabilem. Ut communicabilem, per modum abstractionis per nomen essentiar, et per modum concretionis per nomen substatiae; ut incommunicabilem vero, vel ut distinguibilem per nomen hypostasis; vel notabiliter et perfecte, et sic persona. Vel aliter, scilicet ut distinctam qualitercumque, et sic hypostasis; vel notabiliter et perfecte, et sic persona. Exempla horum quatuor sunt in creatura: humanitas, homo, aliquis homo, Petrus; primum essentiam, secundum substantiam, tertium hypostasim, et quartum personam dicit» (S. Buenaventura, Brevil., p. 1, c. 4: V, 213). Sigue la doctrina de A. de Hales en Summa Helensis, n. 395: I, 583.

38 S. Buenaventura, M. Trin., q. 2, a. 2 concl: V, 65.

39 Manzano, I. G., «Concepto de persona humana», o. c., 399.

40 «Ad illud quod obiicitur, quod persona auto dicitur secundum substantiam, aut secundum relationem etc.; dicendum, qud persona nihil est quam «hypostasis proprietate distincta», et edio dicitur secundum relationem intrinsecam et secundum substantiam non communen, sed propriam» (S. Buenaventura, M. Trin., q. 2, a. 2 concl. ad opp. 9: V, 66-67).
} 
El equilibrio conceptual de sustancia y relación de la persona, implica la singularidad. La persona es naturaleza «in atomo», se distingue por su singularidad, y es ahí donde aparece la tercera parte de la definición que hemos señalado de persona: la dignidad, pues, en efecto, la dignidad es una cualidad necesaria para poder aplicar el concepto de persona. ${ }^{41}$ Persona implica, por lo tanto, individualidad en cuanto que es indivisible (in atomo) y distinto de los demás seres. De este modo, la identidad personal es individualidad lo que supone singularidad, incomunicabilidad y dignidad supereminente. ${ }^{42}$ Las dos primeras notas unidas de individualidad - singularidad e incomunicabilidad-implican lo propio y específico de la persona y le diferencia de la hipóstasis. ${ }^{43}$

En fin, el maestro franciscano realiza así una profundización metafísica del concepto de persona aprovechando el incremento de significación que la expresión había experimentado desde los esquemas teológicos, en términos que algunos han calificado de «trasgresión metafórica», ${ }^{44}$ pero que aquí juzgamos como una oportuna hermenéutica metafísica.

\section{LA PERSONA HUMANA}

Lo que hemos dicho hasta ahora responde a la pregunta sobre el concepto de persona y su conveniencia a la hora de aplicarlo a la Trinidad divina, de quien se dice adecuadamente. Pero lo que nos compete aquí es la cuestión sobre la persona humana, por lo tanto, ¿es aplicable la noción de «persona» al hombre? ${ }^{45}$

En primer lugar, tenemos que el nombre persona definido como está, debería, en principio, aplicarse a cualquier realidad que sea capaz de dignidad. Y la propiedad distinta de la dignidad (supereminente) es una propiedad esencial que solo puede hallarse, descubrirse, en el individuo de la creatura nobilísima, es decir dotada de naturaleza racional, ya que la racionalidad es su propiedad más noble. ${ }^{46}$ Consecuentemente, del hombre, que es racional, cabe decirse que puede soportar la categoría de persona. Además, si persona hace referencia a la naturaleza ha de emplearse de modo semejante a cualquier naturaleza que soporte dignidad.

Ahora bien, en principio parece que se puede decir persona a los seres mientras cumplan la característica de la dignidad, es decir una constitución racional, pero surge la interpelación: ¿cómo se puede decir el mismo nombre de persona para designar a seres tan diversos como son los seres creados e increados? ${ }^{47}$

Como hemos dicho, la distancia ontológica entre Dios creador y la creatura está separada por el hiato insalvable de su propia vanidad. No se puede usar el mismo concepto en realidades que no tienen nada en común con cargas ontológicas sumamente distintas. Además, si la persona se dice de la sustancia es un hecho que Creador y creatura no son realidades que comparten sustancia. Decir persona de modo unívoco a Creador y creatura implica tratarlos del mismo modo, pero en sí ambos no participan en nada. Incluso el problema de la relación naturaleza y persona cambia, mientras que la cuestión de las personas en Dios se plantea en uni-

41 S. Buenaventura, III Sent. d. 5, a. 2, q. 2 ad. 1-2: III, 132. «Hoc enim nomen persona suntum est a dignioribus» (S. Buenaventura, Ibíd., d. 5, a. 2, q. 2 concl.: III, 133).

42 «(...) individuum in notificatione personae triplicem importat distinctionem, videlicet singularis, incommunicabilitatis et superemienetis dignitatis» (l.c.).

43 Cf. S. Buenaventura, I Sent. d. 23, a. 1, q. 3 concl.: I, 409.

44 Melchiorre, V., «Per un'ermeneutica della persona», en Pavan, A. y Milano, A. (eds.), Persona e personalismi, Nápoles, Dehoniane, 1987, p. 289.

45 S. Buenaventura, I Sent., d. 25, a. 2, q. 2: I, 444-445.

46 S. Buenaventura, III Sent., d. 5, a. 2, q. 2 concl.: III, 133.

47 «Utrum nomen personae, dictum de creatis et increatis personis, sit commune univocum» (S. Buenaventura, I Sent., d. 25, a. 2, q. 2: I, 444). 
dad de naturaleza y pluralidad de personas, en el hombre hablamos de unidad personal y dualidad de naturalezas. De este modo, tanto la naturaleza intelectual como la hipóstasis, la distinción y las propiedades son diferentes en Dios y en las creaturas. ${ }^{48}$

La solución que adopta es la analogía del ser, ${ }^{49}$ pues la analogía indica la relación entre dos términos que no comparten una naturaleza común según la semejanza de proporción; ${ }^{50}$ de este modo se sitúa entre el puro equívoco y el unívoco. ${ }^{51}$ Se aplica el nombre del ser al Creador y a la creatura no como a iguales, de forma unívoca, sino según la analogía, pues esta dice algo de alguien, siempre que ocurre una semejanza, como es el caso de la persona creada e increada y por lo tanto no es posible tampoco el equívoco. ${ }^{52}$ Ahora bien, la semejanza se realiza, como hemos dicho, en razón de una similitud proporcional y no en base a una unidad de naturalezas, pues opera una diferencia ontológica insalvable entre Dios y el hombre. Si la persona se aplica a lo creado y lo increado, se realiza por comparación de las realidades de naturalezas diversas, pues frente a la simplicidad de la naturaleza divina, la persona humana lo es de una sustancia compuesta. La persona en Dios es de mayor nobleza que en los hombres. Por lo tanto, existen modos distintos de ser persona según sea en Dios o en el hombre (así Cristo es persona en cuanto Dios y persona en cuanto hombre).$^{53}$ Persona se apunta primero de las personas creadas según el nombre, pero según la cosa nombrada primero se dice de las personas increadas. De este modo, san Buenaventura realiza una dependencia del ser humano creado, del ser en el que sustenta su ser persona. Además, la propia posición de personas realiza la diferenciación.

La persona analogada en el hombre es el supuesto de la distinción de la propiedad. ¿Cómo es posible en el hombre? La pregunta sobre la distinción personal en el hombre (compuesto sustancial de materia y forma $)^{54}$ supone la cuestión sobre la individuación. La persona humana implica la diferencia y distinción propia de la persona, ello significa que el hombre está individualizado, hay una similitud entre las propiedades individuales y personales. Individualidad y personalidad no son dos momentos accidentales del hombre, de modo que uno sea individuo y luego persona como es niño y después adulto, sino que el ser humano comprende un principio intrínseco: son propiedades sustanciales. ${ }^{55}$

Cuestionarse la individuación nos lleva a ver cómo se involucran en el proceso los coprincipios de la naturaleza sustancial presentes en el hombre: la materia y la forma. Así para el Doctor Seráfico, en la medida que la distinción personal dice singularidad, o individuación, surge de dos principios. ${ }^{56}$ Sin negar rotundamente (sino tomados como opinión) ni a Aristóteles (Buenaventura no ve claro que la materia pueda ser el principio y causa principal de la distinción haciendo así que la forma sea dependiente totalmente de la materia) ni a Averroes (para quien siendo la materia común a todos los seres, la individuación reside en la forma, de modo que las formas están en la materia: «posee potencialmente en sí misma forma, aunque de manera intrínseca»), ${ }^{57}$ san Buenaventura afirma que la individuación surge de la actualidad de la unión de la materia con la forma, de modo que ambos coprincipios presentes en el hombre se apropian mutuamente. El individuo es hoc quod, indicando el ser que es, lo que es. Si reali-

48 Ibíd., d. 25, a. 2, q. 2: I, 445.

49 Cf. Berti, E., «Il concetto di analogia in San Bonaventura», en Doctor Seraphicus, 32 (1985), pp. 11-21.

50 I Sent., d. 1, dub V: I, 43

51 «(..) quod [analogo] est medium inter puer aequivocum et univocum» (Ibíd., d. 29, a. 1, q. 2 concl.: I, 511).

52 Ibíd., d. 25, a. 2, q. 2: I, 445.

53 S. Buenaventura, III Sent., d. 10, a. 1, q. 3 concl.: III, 228.

54 «Communicat autem quodam modo potentia intellectiva operationem suam sorpori coniuncto, cum communicet ei esse, et ex eis fiat unum per essentiam, non solum in quantum anina est vegetabilis et sensibilis, sed etiam in quantum rationalis» (S. Buenaventura, II Sent., d. 25, p. 2, a. un., q. 4 concl.: II, 622).

55 Cf. S. Buenaventura, II Sent., d. 3, p. 1, q. 2.: II, 105-107.

56 Ibíd., d. 3, p. 1, q. 3 concl.: II, 109.

57 Ramón Guerrero, R., Filosofías árabe y judía, Madrid, Síntesis, 2001, p. 235. 
zamos un análisis desde el entendimiento podemos decir que el responsable de la indicación del ser humano (hoc), lo que hace de modo primordial que el hombre se manifieste y se haga presente en el mundo espacio-temporal, es su principio material. En cuanto a lo que es (quod), como hombre individualizado, el responsable lo encontramos en la forma. El individuo tiene ser (el acto de existir descansa en la forma de la materia) y existir (que lo da la materia formal), por lo que en el hombre (en las creaturas), el individuo surge de los dos principios, que no olvidemos son indisolubles.

Por lo tanto, la persona se distingue por su distinción en las propiedades, su ser supuesto y su hipóstasis. De este modo, toda realidad sea por sí o analogada tiene estas características, que comunes en lo increado o lo creado, hace de la persona algo totalmente distinto e incomunicado, pues individuación implica incomunicación. La persona es singular (en cuanto que es una unidad de la distinción de sus propiedades) y, de modo especial, en ella reside la dignidad del hombre, su diferencia más original.

El supuesto personal del hombre, lo que es, implica su dignidad y nobleza, ${ }^{58}$ y es que también la diferencia personal se dice singularidad y dignidad. La singularidad en relación íntima a la individuación es un principio de unificación de los principios de lo que es (quod est). La dignidad deriva, sin embargo, en razón de la forma; y es una característica principal de diferencia personal en los hombres y en los ángeles respecto de las demás criaturas creadas por Dios. Como señala L. Iammarrone la persona dota un orden del ser que hace que se distinga de las simples hipóstasis que dotadas de una propiedad común no les dota de ningún grado eminente. «Por este motivo, —explica Iammarrone- el término persona se suele predicar de aquellos entes que están en posesión de la naturaleza racional, que, por su nobleza en el orden del ser, se distinguen de todos los otros entes de naturaleza inferior».59

El principio espiritual, el alma (la forma, principio de acción y conocimiento) es la cualidad que fundamenta la nobleza y dignidad personal humana, en cuanto que tiene capacidad no solo de entender la realidad creada que le rodea, sino también «la esencia creadora». ${ }^{60} \mathrm{Y}$ aunque el hombre posea un principio corporal, el alma opera la reducción hacia lo superior y capacita al hombre a participar de la bienaventuranza y felicidad divinas en un acto reductivo y comunicativo. ${ }^{61} \mathrm{El}$ alma hace al hombre capaz de Dios (capax Dei $)^{62}$ al estar «hecha a imagen de la Trinidad». ${ }^{63}$ Reduciendo y participando de Dios en cuanto imagen, el alma racional posibilita la semejanza con Dios. La dignidad distintiva de la persona humana a través del alma racional dota de contenido real la analogía en virtud del ejemplarismo metafísico, trazando nuevamente sus fuentes más queridas: el neoplatonismo en la lectura latina, agustinista (capax Dei), ${ }^{64}$ y griega, dionisiana (Bonum diffusisvum

58 S. Buenaventura, II Sent., d. 3, p. 1, q. 3 concl.: II, 110.

59 Iammarrone, L., «La Trinidad», o. c., 89. Cf. S. Buenaventura, I Sent., d. 23, a. 1, q. 3: I, 409-410.

60 S. Buenaventura, Brevil., p. 2, c. 9, n. 1: V, 226.

61 «Deus enim universa propter semetipsum operatus est, ita quod, cum sit summa potestas et maiestas, fecit omnia ad laudem; cum sit summa bonitas, fecit omnia ad sui communicationem» (S. Buenaventura, I Sent., d. 16, a. 1 , q. 1 concl.: II, 394).

62 «(..) eo quod 'capax Dei est et participes esse potest', inmediate ordinatur in ipsum» (Ibíd., d. 16, a. 1, q. 1 concl.: II, 395). Cf. Szabo, T., De SS. Trinitate in creaturis refulgente doctrina S. Bonaventurae, Roma, Herder, 1955, pp. 65-85; Langevin, G., "Capax Dei». La crétaure intellectuelle et l' intimité de Dieu, Bruges-Paris, Desclee De Brouwer, 1966; Mourien, T., «Capacitas», en Bougerol, J. -G. (dir.), Lexique saint Bonaventure, Paris, Ed. Franciscaines, 1969, p. 29; Mathieu, L., «La Trinité», o. c., pp. 236-242.

63 S. Buenaventura, I Sent., d. 16, a. 1, q. 1 concl.: II, 394.

64 «Eo quippe ipso imago ejus est, quo ejus capax est, ejusque particeps esse potest» (S. Agustín, De Trinitate, c. 8, n. 11, PL 42, p. 1044). 
sui). ${ }^{65}$ De este modo, el alma, fundamentada en la imagen, que da capacidad al hombre de poder elevarlo más en nobleza, en razón de la semejanza, establece al principio distinto y fundamenta la relación personal. La materia del ser humano (vestigio), como todo principio corporal puede referirse a Dios como a principio creador; pero lo que puede hacer que se establezca una relación como a objeto motivo y como a don inhabitante, es el alma. ${ }^{66}$

El hombre adquiere en la persona, tanto su dignidad personal, su definición, como su singularidad y su carácter de relación. El ser persona del hombre le confiere al hombre el ser uno, singular, con capacidad de existir y operar por su propia cuenta, independientemente de otros sujetos. ${ }^{67}$ Pero ello no lleva al individualismo, sino que en la singularidad individual de la persona se puede aplicar unívocamente el ser personal a todos los seres que comparten su ser creados o increados. La constitución personal en el hombre es importada del origen (ex principiis

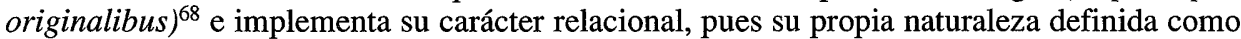
carencia exige la relación con quien le dota de existencia. La aplicación de relación en el hombre, ser creado, no es la misma que en Dios, pues a diferencia que Dios que se identifica con la realidad sustancial del sujeto que le precede en el ser, en el hombre no puede operarse dicha identificación. La relación tiene un papel constitutivo en las personas divinas que en el hombre no posee. ${ }^{69}$ De este modo, el hombre es en sí un misterio que se abre, se relaciona y, a la vez, se funda en el misterio de quien es analogado. La estructura de la persona humana se imbrica de un modo esencial en su más honda profundidad, en cuanto que se vincula al exceso del misterio personal del misterio por excelencia: el Trinitario.

El pensamiento bonaventuriano realiza una profundización antropológica del ejemplarismo al penetrar el concepto de persona al que nos referimos en el primer apartado; del mismo modo, que la reflexión de la persona humana penetra el ejemplarismo e inspira toda la antropología metafísico-simbólica. Una simbiosis que se significa en las propiedades personales: la singularidad e incomunicabilidad de la persona queda abierta al Creador, pues la metafísica ejemplar es un sistema de relaciones entre Creador y creatura.

Manuel Lázaro Pulido e-mail: mlazarop@buenaventura.jazztel.es

65 Pseudo-Dionisio, De divinis nominibus, c.4, n. 1, PG 3, p. 693b. Cf. De coelesti hierarchia, c.4, n. 1, PG 3, p. 177c. El origen de esta expresión descansa en el Pseudo-Dionisio, en concreto, aparece en su obra De divinis nominibus cuando afirma que «en cuanto bien esencial, él (Dios-Bien) extiende su bondad a todo ser». A san Buenaventura le llega esta fórmula de modo indirecto. Conocía, sin duda, los escritos de Felipe el Canciller, Guillermo de Auxerre y Guillermo de Auvergne; pero toma contacto con este axioma, sobre todo, de la mano de Alejandro de Hales. Cf. Muñiz, V., «A propósito del Pseudo-Dionisio en San Buenaventura», en Naturaleza y Gracia, 21 (1974), pp. 173-181.

66 S. Buenaventura., Brevil., p.2, c.12: V, 230.

67 Cf. S. Buenaventura, I Sent., d. 23, a. 1., q. 1: I, 405.

68 Cf. Ibíd., d. 34, a. un., q. 1: I, 586-588.

69 Cf. Iammarrone, L., «La Trinidad», o. c., 93. 